\title{
AVALIAÇÃO DA EXPRESSÃO DOS GENES ABCB1, ABCC1, ABCG2 EM PACIENTES COM LÚPUS ERITEMATOSO SISTÊMICO
}

\author{
M. F. D. SOUZA ${ }^{1}$, H. L. S NETA ${ }^{1}$, H. A. MARIZ ${ }^{2}$, M. J. B. M. RÊGO ${ }^{1}$, M. C. PEREIRA ${ }^{1}$, \\ I. R. PITTA ${ }^{1}$, C. D. L. MARQUES ${ }^{2}$, A. L. B. P. DUARTE ${ }^{2}$, M. G. R. PITTA ${ }^{1}$ \\ ${ }^{1}$ Laboratório de Imunomodulação e Novas Abordagens Terapêuticas/ LINAT, Núcleo de \\ Pesquisa em Inovação Terapêutica Suely Galdino - NUPIT SG, Universidade Federal de \\ Pernambuco - UFPE \\ ${ }^{2}$ Hospital das Clínicas de Pernambuco, UFPE, Recife-PE. \\ E-mail para contato: deodatomariadefatima@gmail.com
}

\begin{abstract}
RESUMO - O objetivo desse estudo foi avaliar a expressão gênica de transportadores $A B C B 1, A B C C 1$ e ABCG2 pertencentes a superfamília de transportadores $A B C$ (do inglês, ATP binding cassete)e relacionadas ao fenótipo Multidrug Resistance - MDR em pacientes com LES frente às manifestações clínicas da doença. Foram selecionados 28 pacientes (26 mulheres e 2 homens) com idade média de 35,1 $\pm 10,9$ anos e 15 mulheres sadias (grupo controle) com idade média de $34.4 \pm 11.5$. Os pacientes foram recrutados do serviço de Reumatologia do Hospital das Clínicas da UFPE e preencheram os critérios de classificação do American College of Rheumatology para o LES. A expressão gênica foi avaliada pela técnica de reação em cadeia da polimerase em tempo real (RTq-PCR) com sondas TaqMan ${ }^{\circledR}$. A expressão gênica de ABCB1, ABCC1, $A B C G 2$ não divergiu entre pacientes e voluntários sadios ( $p \geq 5$ ). Não foram detectadas correlações entre os parâmetros clínicos avaliados (SLEDAI, anti$D N A$, nefrite, proteínas do complemento e proteinúria) e a expressão dos genes estudados $(p>0,05)$. No entanto, através de análises de regressão linear múltipla, foram observadas fortes correlações positivas entre a expressão dos genes $A B C B 1$, ABCC1, ABCG2 $(p \leq 0,002)$ de pacientes com LES. Nossos dados indicam correlações entre a expressão dos transportadores que precisam ser investigadas em um maior número de paciente. Estes dados podem promover novos estudos que visem uma melhor compreensão da doença na perspectiva da reversão do fenótipo $M D R$.
\end{abstract}

Palavras-chave: Lúpus eritematoso sistêmico, transportadores ABC, Multidrug Resistance.

ABSTRACT - The objective of this study was to evaluate the gene expression of $A B C B 1, A B C C 1$ and $A B C G 2$ transporters belonging to the ABC superfamily (ATP binding cassette) and related to the Multidrug Resistance (MDR) phenotype in patients with SLE in the clinical manifestations of the disease. 28 patients (26 women and 2 men) with a mean age of $35.1 \pm 10.9$ years and 15 healthy women (control group) with a mean age of $34.4 \pm 11.5$ were selected Patients were 
recruited from the Rheumatology Service of the UFPE Hospital das Clinicas and met the American College of Rheumatology classification criteria for SLE. Gene expression was assessed by the real-time polymerase chain reaction (RTq-PCR) technique with TaqMan ${ }^{\circledR}$ probes. The gene expression of $A B C B 1, A B C C 1, A B C G 2$ did not differ between patients and healthy volunteers $(p>5)$. Correlations between clinical parameters (SLEDAI, anti-DNA, nephritis, complement proteins and proteinuria) and expression of the genes studied were not detected ( $p>0.05)$. However, through multiple linear regression analyzes, strong positive correlations were observed between expression of $A B C B 1, A B C C 1, A B C G 2(p<0.002)$ genes of SLE patients. Our data indicate correlations between the expression of the transporters that need to be investigated in a larger number of patients. These data may promote further studies aiming at a better understanding of the disease in view of the reversal of the MDR phenotype.

Keywords: Systemic lupus erythematosus, ABC transporters, Multidrug Resistance.

\section{INTRODUÇÃO}

O lúpus eritematoso sistêmico (LES) é uma doença inflamatória autoimune de etiologia pouco esclarecida e elevado potencial mórbido (SILVA et al., 2014). Essa doença é caracterizada principalmente pela ativação de linfócitos $\mathrm{T}$ e $\mathrm{B}$ autorreativos, formação de auto-anticorpos, imunocomplexos circulantes, e atuação de diversos mediadores da inflamação (CHUN et al., 2007; FOSTER, 2007; OHL; TENBROCK, 2011; PATHAK; MOHAN, 2011).

Na prática clínica, o tratamento depende das manifestações da doença, sendo frequentemente utilizados corticosteróides, antimaláricos e imunossupressores. No entanto, muitas vezes os pacientes tornam-se refratários a estes tratamentos convencionais. Em particular, isto reduz as chances de controle da atividade da doença e aumenta o risco de morte (BAZSÓ et al., 2015; PICCHIANTI-DIAMANTI et al., 2014; TSUJIMURA et al., 2007).

A superexpressão de transportadores ABCs do inglês, ATP binding cassette, tem emergindo como uma importante causa de resistência a múltiplas drogas (MDR) durante a quimioterapia de doenças autoimunes, tais como artrite reumatóide, psoríase e LES (PICCHIANTI-DIAMANTI et al., 2014; TSUJIMURA et al., 2007). Atualmente, pouco se sabe sobre o papel dos transportadores ABCs no insucesso da terapêutica do LES. Portanto, o presente estudo visou investigar os principais transportadores $\mathrm{ABCs}$ envolvidos no fenótipo MDR (ABCB1, ABCC1 e ABCG2) frente as manifestações clínicas do LES.

\section{MATERIAIS E MÉTODOS}

\subsection{PACIENTES}

Os pacientes foram atendidos no Serviço de Reumatologia do Hospital das Clínicas (HC) da Universidade Federal de Pernambuco. Foram incluídos neste estudo 26 mulheres e 2 homens (20 a 65 anos), com diagnóstico de LES de acordo com o American College of Rheumatology (ACR). O grupo controle foi composto por 15 mulheres de 
mesma faixa etária, sem LES e sem diagnóstico para imunodeficiências. A atividade da doença foi mensurada utilizando o Systemic Lupus Erythematosus Disease Activity Index (SLEDAI) (Gladman et al, 2002). Todos os indivíduos assinaram o termo de consentimento livre e esclarecido (TCLE) (CEP/CCS/UFPE No 145/09).

\subsection{SEPARAÇÃO DAS CÉlULAS MONONUClEARES DO SANGUE PERIFÉRICO (PBMCs)}

As PBMCs foram obtidas a partir de amostras de sangue periférico dos pacientes com LES e dos voluntários sadios, utilizando a técnica de centrifugação com Ficoll-Paque PLUS (GE Healthcare).

\subsection{QUANTIFICAÇÃO DA EXPRESSÃO GÊNICA}

As PBMCs foram resuspendidas em trizol (Invitrogen) e o RNA foi extraído pelo método fenol-clorofórmico. Em seguida, o RNA foi utilizado para a formação do DNA complementar (cDNA) via transcriptase reversa com o Kit: High-capacity cDNA archive Kit $2 X$ (Applied Biosystems) e a quantificação do cDNA foi feita por PCR quantitativa, utilizando o método TaqMan (Applied Biosystems) no aparelho 7900 HT Fast real time PCR (Applied Biosystems). O gene 18S foi utilizado como o controle endógeno. A expressão relativa do RNA de ABCB1, ABCC1 e ABCG2 foi calculada pelo método 2$\triangle \triangle \mathrm{Ct}$ (SAMBROOK \& GREEN, 2012).

\subsection{ANÁLISES ESTATÍSTICAS}

Para a análise de normalidade dos dados foi aplicado o teste de normalidade Shapiro-Wilk. As análises estatísticas foram realizadas por meio de comparações univariadas, utilizando teste não paramétrico (Mann-Whitney). Para a análise de correlação de dados não paramétricos foi utilizado o teste de Spearman. Os dados foram representados graficamente pelo software GraphPad Prism ${ }^{\circledR}$ versão 6.0. Os resultados foram mostrados considerando o valor da mediana, mínimo e máximo, sendo $\mathrm{P}<0,05$ considerado como significativo.

\section{RESULTADOS E DISCUSSÃO}

Dos 28 pacientes com LES incluídos neste estudo, 42,9\% estavam com a doença em remissão e 57,1\% em atividade, conforme apresentado na tabela 1 . 
Tabela 1. Caracterização clínica e demográfica dos pacientes com LES recrutados para este estudo.

\begin{tabular}{cc}
\hline Número de Pacientes & $\mathrm{N}=28$ \\
\hline $\begin{array}{c}\text { Idade (anos), } \\
\text { Média } \pm \text { dp }\end{array}$ & $35,1 \pm 10,9(20-65)$ \\
Sexo, N (\%) & \\
Masculino & $2(7.1)$ \\
Feminino & $26(92.9)$ \\
Anti-DNA, N (\%) & \\
Positivo & $8(28.6)$ \\
Negativo & $20(71.4)$ \\
Complemento, N (\%) & \\
Baixo & \\
Normal & $17(60.7)$ \\
& $11(39.3)$ \\
Atividade da doença & \\
(SLEDAI), N (\%) & \\
$<6$ & $11(39.3)$ \\
$\geq 6$ & $17(60.7)$ \\
Anativa &
\end{tabular}

De acordo com o gráfico 1, não foram encontradas variações significativas entre a expressão gênica relativa das proteínas $\mathrm{ABCB} 1, \mathrm{ABCC} 1$ e $\mathrm{ABCG} 2$ de pacientes e voluntários sadios $(\mathrm{p}>0,05)$ (Gráfico 1).

Gráfico 1. Expressão gênica relativa de $\mathrm{ABCB} 1, \mathrm{ABCC} 1$ e $\mathrm{ABCG} 2$ em pacientes com LES e indivíduos sadios.

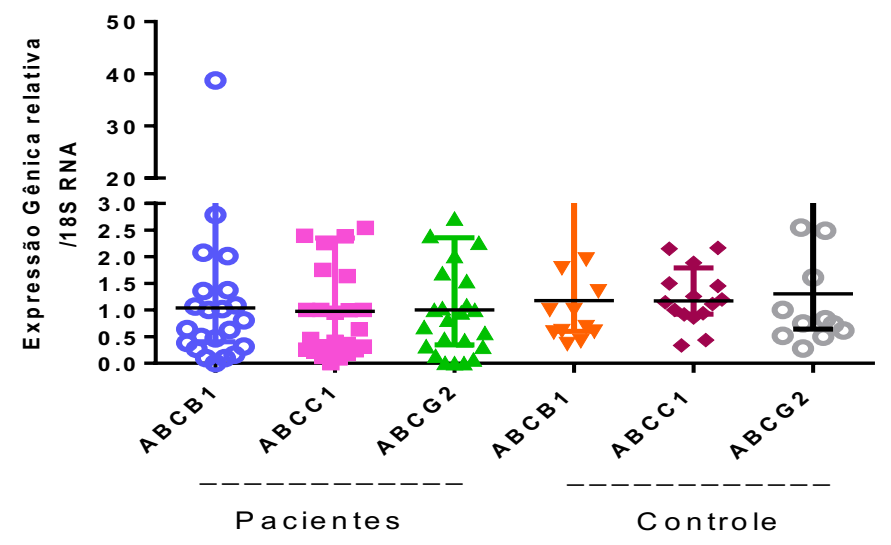


A expressão dos transportadores $\mathrm{ABC}$ foi investigada em relação à atividade da doença. Não houve correlação significativa entre o aumento do SLEDAI e a expressão de ABCB1 $(p=049), \operatorname{ABCC} 1(p=0,58)$ ou ABCG2 $(p=0,90)$. Da mesma maneira, análises adicionais referentes ao acometimento de nefrite, redução de proteínas do complemento, formação de anti-DNA ou demais complicações clínicas da doença em relação à expressão de $\mathrm{ABCB} 1, \mathrm{ABCC} 1$ ou $\mathrm{ABCG} 2$ também não foram significativas $(\mathrm{p}>0,05)$.

Embora não tenham sido significativas as divergências entre a expressão dos genes ABCB1, ABCC1, ABCG2 de pacientes com LES e voluntários sadios, as análises de regressão linear múltipla apresentaram uma correlação positiva entre a expressão dos genes $\mathrm{ABCB} 1, \mathrm{ABCC} 1, \mathrm{ABG} 2$ nos pacientes $(\mathrm{p}<0,0001)$ (Tabela 2).

Tabela 2. Correlação entre a expressão dos genes $\mathrm{ABCB} 1, \mathrm{ABCC} 1, \mathrm{ABCG} 2$ nos pacientes com LES.

\begin{tabular}{llll}
\hline & ABCB1 & ABCC1 & ABCG2 \\
\hline \multirow{2}{*}{ ABCB1 } & & $\mathrm{p}<0,0001$ & $\mathrm{p}<0,0001$ \\
& & $\mathrm{r}=0,803$ & $\mathrm{r}=0,679$ \\
& $\mathrm{p}<0,0001$ & & $\mathrm{p}<0,0001$ \\
ABCC1 & $\mathrm{r}=0,803$ & & $\mathrm{r}=0,804$ \\
& & & \\
\hline \multirow{2}{*}{ ABCG2 } & $\mathrm{p}=<0,0001$ & $\mathrm{p}<0,0001$ & \\
& $\mathrm{r}=0,679$ & $\mathrm{r}=0,804$ & \\
\hline
\end{tabular}

Ainda são escassos os estudos que investigam transportadores $\mathrm{ABC}$ no LES. Embora não tenham sido encontradas correlações clínicas significativas, nosso conjunto de dados indica fortes correlações entre todas as proteínas estudadas. Portanto, nossos dados abrem perspectivas para novas investigações que visem à compreensão do envolvimento destas proteínas no desenvolvimento da doença.

\section{CONCLUSÃO}

Nossos resultados demostraram que pacientes e voluntários sadios não apresentam diferenças significativas na expressão dos transportadores $\mathrm{ABCB} 1, \mathrm{ABCC} 1$ e ABCG2. Não foram encontradas correlações significativas entre a expressão dos genes avaliados e os parâmetros clínicos analisados (SLEDAI, anti-DNA, nefrite, proteínas do complemento e proteinúria). No entanto, foram encontradas fortes correlações positivas entre a expressão dos genes avaliados as quais precisam ser investigados em um maior número de pacientes. Estes dados podem promover novos estudos que visem uma melhor compreensão da doença e a busca de novas estratégias terapêuticas na perspectiva da reversão do fenótipo MDR. 


\section{REFERÊNCIAS}

BAZSÓ, A. et al. Autoimmunity Reviews The importance of glucocorticoid receptors in systemic lupus erythaematosus . A systematic review. Autoimmunity Reviews, 2015.

CHUN, H.-Y. et al. Cytokine IL-6 and IL-10 as Biomarkers in Systemic Lupus Erythematosus. Journal of Clinical Immunology, v. 27, n. 5, p. 461-466, 2007.

FOSTER, M. H. T cells and B cells in Lupus Nephritis. NIH Public Access, v. 27, n. 1, p. 47-58, 2007.

OHL, K.; TENBROCK, K. Inflammatory Cytokines in Systemic Lupus Erythematosus. Journal of Biomedicine and Biotechnology, 2011.

PATHAK, S.; MOHAN, C. Cellular and molecular pathogenesis of systemic lupus erythematosus: lessons from animal models. Arthritis Research \& Therapy, v. 13, n. 5, p. 241, 2011.

PICCHIANTI-DIAMANTI, A. et al. P-Glycoprotein and Drug Resistance in Systemic Autoimmune Diseases. International Journal of Molecular Sciences, p. 4965-4976, 2014.

SAMBROOK J, GREEN M. Molecular Cloning: A Laboratory Manual, $4^{\text {a }}$ ed., Cold Spring Harbor Laboratory Press, 2012.

SILVA, H. D. A. et al. Interferon gamma and Interleukin 10 polymorphisms in Brazilian patients with systemic lupus erythematosus. Molecular Biology Reports, v. 41, n. 4, p. 2493-2500, 2014.

TSUJIMURA, S. et al. Relevance of multidrug resistance 1 and P-glycoprotein to drug resistance in patients with systemic lupus erythematosus. Histology and Histopathology, v. 22, p. 465-468, 2007.

\section{AGRADECIMENTOS}

PIBITI-UFPE, CNPq, INCT-if, CAPES. 